\title{
Clinical risk-scoring algorithm to forecast scrub typhus severity
}

This article was published in the following Dove Press journal:

Risk Management and Healthcare Policy

12 December 2013

Number of times this article has been viewed

\author{
Pamornsri Sriwongpan ${ }^{1,2}$ \\ Pornsuda Krittigamas ${ }^{3}$ \\ Hutsaya Tantipong ${ }^{4}$ \\ Jayanton Patumanond ${ }^{5}$ \\ Chamaiporn Tawichasri ${ }^{6}$ \\ Sirianong Namwongprom ${ }^{1,7}$ \\ 'Clinical Epidemiology Program, \\ Chiang Mai University, Chiang Mai, \\ Thailand; ${ }^{2}$ Department of Social \\ Medicine, Chiangrai Prachanukroh \\ Hospital, Chiang Rai, Thailand; \\ ${ }^{3}$ Department of General Pediatrics, \\ Nakornping Hospital, Chiang Mai, \\ Thailand; ${ }^{4}$ Department of Medicine, \\ Chonburi Hospital, Chonburi, \\ Thailand; ${ }^{5}$ Clinical Epidemiology \\ Program, Thammasat University, \\ Bangkok, Thailand; ${ }^{6} \mathrm{Clinical}$ \\ Epidemiology Society at Chiang Mai, \\ Chiang Mai, Thailand; ${ }^{7}$ Department \\ of Radiology, Chiang Mai University, \\ Chiang Mai, Thailand
}

Correspondence: Sirianong Namwongprom

Department of Radiology, Faculty of Medicine, Chiang Mai University,

Chiang Mai 50200,Thailand

Tel +66 53945458

Fax +66 53945476

Email snamwong@med.cmu.ac.th
Purpose: To develop a simple risk-scoring system to forecast scrub typhus severity.

Patients and methods: Seven years' retrospective data of patients diagnosed with scrub typhus from two university-affiliated hospitals in the north of Thailand were analyzed. Patients were categorized into three severity groups: nonsevere, severe, and dead. Predictors for severity were analyzed under multivariable ordinal continuation ratio logistic regression. Significant coefficients were transformed into item score and summed to total scores.

Results: Predictors of scrub typhus severity were age $>15$ years, (odds ratio $[\mathrm{OR}]=4.09$ ), pulse rate $>100$ /minute (OR 3.19), crepitation (OR 2.97), serum aspartate aminotransferase $>160 \mathrm{IU} / \mathrm{L}(\mathrm{OR} 2.89)$, serum albumin $\leq 3.0 \mathrm{~g} / \mathrm{dL}$ (OR 4.69), and serum creatinine $>1.4 \mathrm{mg} / \mathrm{dL}$ (OR 8.19). The scores which ranged from 0 to 16 , classified patients into three risk levels: non-severe (score $\leq 5, \mathrm{n}=278,52.8 \%$ ), severe (score 6-9, $\mathrm{n}=143,27.2 \%$ ), and fatal (score $\geq 10, n=105,20.0 \%$ ). Exact severity classification was obtained in $68.3 \%$ of cases. Underestimations of $5.9 \%$ and overestimations of $25.8 \%$ were clinically acceptable.

Conclusion: The derived scrub typhus severity score classified patients into their severity levels with high levels of prediction, with clinically acceptable under- and overestimations. This classification may assist clinicians in patient prognostication, investigation, and management. The scoring algorithm should be validated by independent data before adoption into routine clinical practice.

Keywords: severe scrub typhus, risk-scoring system, clinical prediction rule, prognostic predictors

\section{Introduction}

Scrub typhus is one of the common acute febrile illnesses encountered in tropical countries, including Thailand. ${ }^{1,2}$ The infection often causes vasculitis and multipleorgan failure. ${ }^{3,4}$ Patients with such complications usually have poor prognoses that may end with death, especially those with delayed diagnosis and treatment. Systemic complications commonly reported as causes of death include respiratory involvement $(15 \%-36 \%),{ }^{5-7}$ cardiovascular involvement $(2 \%-34 \%),{ }^{5,6,8,9}$ renal involvement $(9 \%-20 \%),{ }^{6,9-11}$ hepatic involvement $(4 \%-31 \%),{ }^{8,9}$ central nervous system involvement $(4 \%-23 \%),{ }^{8,12}$ or multiple-organ involvement $(11.9 \%) .{ }^{13}$ Mortality is reported in up to $30 \%$ of cases. ${ }^{4,14}$

Clinical characteristics for prognostication of scrub typhus severity and death have been the subject of many studies. These characteristics may include any combinations of the following systems: respiratory system - dyspnea, crepitation, and abnormal chest film; cardiovascular system - septic shock; hepatobiliary system - serum 
albumin $\leq 3 \mathrm{~g} / \mathrm{dL}$, bilirubin $>1.5 \mathrm{mg} / \mathrm{dL}$, and more than twofold increase in aspartate aminotransferase (AST); and kidney system - serum creatinine $>1.4 \mathrm{mg} / \mathrm{dL}$ and positive urine albumin. ${ }^{12,15-17}$ Early detection of these characteristics might be used to assist clinical guidelines for patient management.

Clinical prediction rules to forecast disease severity were developed for a few infectious diseases, such as communityacquired pneumonia. ${ }^{18}$ However, there have been no studies attempting to develop a clinical risk-scoring system to forecast the severity of scrub typhus.

The present study was conducted to develop a simple clinical risk-scoring algorithm to predict the severity of scrub typhus in patients suspected of infection. The scoring system may assist routine clinical guidelines to improve patient management.

\section{Patients and methods}

This study was approved by the research ethics committees of the two hospitals in Chiang Mai and Chiang Rai, and the Faculty of Medicine at Chiang Mai University.

\section{Patients}

Retrospective data analysis was conducted at two universityaffiliated general hospitals in the north of Thailand during 2004 and 2010. Patients were professionally diagnosed with scrub typhus, based on the exposure history of disease, presenting with acute fever and at least one of the following signs and symptoms: myalgia, headache, conjunctival injection, cough, profuse sweating, maculopapular rash, and lymphadenopathy, ${ }^{14}$ accompanied by the presence of eschar and/ or positive immunochromatographic test for scrub typhus. Patients were classified into three groups: 1) nonsevere patients without any complications; 2) severe - patients with severe complications; and 3) dead - those who died in hospital from scrub typhus. Patients undergoing any intervention trials during the same period were excluded from data analysis.

\section{Definitions of severe scrub typhus}

Severe scrub typhus was operationally defined as patients who presented with involvement of at least one of the following organ systems.

- Cardiovascular system - presence of any of the following:

- Systolic blood pressure less than $90 \mathrm{mmHg}$

- Abnormal cardiac arrhythmia with no previous history of

$\S$ atrial fibrillation (AF),
$\S$ supra ventricular tachycardia (SVT), or

$\S$ frequent premature ventricular tachycardia (PVC)

- Myocarditis: elevated creatine kinase MB above baseline

- Respiratory system: presence of acute respiratory distress syndrome, defined as $\mathrm{PaO}_{2} / \mathrm{FiO}_{2}<200 \mathrm{mmHg}$, with bilateral interstitial infiltration on chest film with normal cardio/thoracic ratio, or no volume overload of central venous pressure from central venous catheter

- Central nervous system - presence of any of the following:

- Glasgow Coma Scale $\leq 12$ without other causes,

- Seizure without other causes, or

- Meningoencephalitis

- Hematology: platelet count $\leq 20,000 / \mathrm{mm}^{3}$

- Urinary tract: presence of acute renal failure, defined as creatinine $\geq 2 \mathrm{mg} / \mathrm{dL}$ or creatinine change $>0.5 \mathrm{mg} / \mathrm{dL} /$ day

- Gastrointestinal and hepatobiliary tract: presence of hepatitis, defined as elevated AST or alanine aminotransferase (ALT) more than fivefold.

\section{Predictor parameters}

Demographic and disease-exposure histories were obtained from medical files recorded at the time of admission: sex, age, underlying diseases (liver cirrhosis, chronic obstructive pulmonary disease, hypertension, diabetes, and human immunodeficiency virus infection), durations of fever.

Signs and symptoms were obtained from chief complaint, history-taking records, and physical examination notes: myalgia, headache, conjunctival injection, cough, maculopapular rash, eschar, lymphadenopathy, abdominal pain, nausea/ vomiting, diarrhea, jaundice, hepatomegaly, splenomegaly, seizure, stiff neck, wheezing, crepitation, dyspnea, respiration rate, and presence of fever.

Hemodynamic profiles were obtained from vital-sign record forms: systolic and diastolic blood pressure and pulse rate. Laboratory profiles were obtained from laboratory investigations requested within 24 hours after admission: hematological, biochemistry, urine analyses.

\section{Data analysis}

Patient clinical characteristics were compared across the three severity groups by nonparametric trend tests across ordered groups. Strong potential predictors were explored by multivariable ordinal continuation ratio logistic regression $(\alpha<0.001)$. The coefficients of significant predictors were transformed into item scores and summed as scrub typhus 
severity scores. The scores were classified into risk levels based on inclining of actual risks. Score-categorized risk levels were compared to criterion risk levels to indicate the score performances.

\section{Results}

A total of 526 patients with document-confirmed professional diagnosis of scrub typhus were retrieved; 215 cases presented with only eschar, 227 cases had positive immunochromatographic test for scrub typhus, and 84 cases had shown both. These patients were classified as nonsevere scrub typhus $(n=357)$, severe scrub typhus $(n=100)$, and dead of scrub typhus ( $\mathrm{n}=69$ ). The majority of patients were males with similar underlying diseases and durations of fever, but were different according to age (Table 1).

Clinical signs and symptoms were similar for myalgia, conjunctival injection, cough, abdominal pain, nausea/ vomiting, maculopapular rash, eschar, hepatomegaly, splenomegaly, and respiration rate. Those that were different were headache, diarrhea, jaundice, lymphadenopathy, seizure, stiff neck, wheezing, crepitation, dyspnea, body temperature, systolic blood pressure, diastolic blood pressure, and pulse rate (Table 1).

Similar laboratory results included hematocrit, hemoglobin, serum globulin, potassium, and chloride. Differences were detected on white blood cell (WBC) count, platelets, neutrophils, lymphocytes, monocytes, total bilirubin, direct bilirubin, albumin, AST, ALT, alkaline phosphatase, blood urea nitrogen creatinine, blood sodium, carbon dioxide, urine albumin levels, and urine sugar (Table 2). There were some missing data, as indicated in the corresponding tables.

\section{Significant predictors}

Multivariable significant $(P<0.001)$ predictors for severity were age $>15$ years (odds ratio [OR] 4.09, 95\% confidence interval [CI] 2.26-7.40), pulse rate $>100 /$ minute (OR 3.19, 95\% CI 1.87-5.43), crepitation (OR 2.97, 95\% CI 1.63-5.39),

Table I Patient profiles $(n=526)$

\begin{tabular}{|c|c|c|c|c|}
\hline Characteristics & $\begin{array}{l}\text { Nonsevere }(n=357) \\
n(\%)\end{array}$ & $\begin{array}{l}\text { Severe }(n=100) \\
n(\%)\end{array}$ & $\begin{array}{l}\text { Dead }(n=69) \\
n(\%)\end{array}$ & $P$-value \\
\hline \multicolumn{5}{|l|}{ Demographics and history } \\
\hline Male & $20 \mathrm{I}(56.3)$ & $55(55.0)$ & $35(50.7)$ & 0.413 \\
\hline Age (years), median (IQR) & $17.0(7.0-42.0)$ & $31.5(\mid 2.5-50.0)$ & $46.0(34.0-64.0)$ & $<0.001$ \\
\hline Underlying diseases & $30(8.4)$ & $14(14.0)$ & $8(11.6)$ & 0.186 \\
\hline Durations of fever (days), median (IQR) & $6(4-7)$ & $7(4-7)$ & $7(4-7)$ & 0.961 \\
\hline \multicolumn{5}{|l|}{ Signs and symptoms } \\
\hline Myalgia & $69(19.3)$ & $23(23.0)$ & I3 (I8.8) & 0.820 \\
\hline Headache & $117(32.8)$ & $29(29.0)$ & $14(20.3)$ & $0.04 I$ \\
\hline Conjunctival injection & $330(92.4)$ & $90(90.0)$ & $64(92.8)$ & 0.823 \\
\hline Cough & $120(33.6)$ & $32(32.0)$ & $17(24.6)$ & 0.172 \\
\hline Maculopapular rash & $324(90.8)$ & $95(95.0)$ & $66(95.7)$ & 0.086 \\
\hline Eschar & $192(53.8)$ & $60(60.0)$ & $38(55.1)$ & 0.557 \\
\hline Lymphadenopathy & $237(66.4)$ & $73(73.0)$ & $63(91.0)$ & $<0.001$ \\
\hline Abdominal pain & $118(33.1)$ & $35(35.0)$ & $25(36.2)$ & 0.565 \\
\hline Nausea/vomiting & $83(23.3)$ & $27(27.0)$ & $12(19.4)$ & 0.544 \\
\hline Diarrhea & $60(16.8)$ & $25(25.0)$ & $17(24.6)$ & 0.047 \\
\hline Jaundice & II (3.1) & $2 \mathrm{I}(2 \mathrm{I} .0)$ & $17(24.6)$ & $<0.001$ \\
\hline Hepatomegaly & $113(31.7)$ & $36(36.0)$ & $14(20.3)$ & 0.198 \\
\hline Splenomegaly & $40(11.2)$ & $9(9.0)$ & $4(5.8)$ & 0.157 \\
\hline Seizure & $4(1.1)$ & $10(10.0)$ & $12(17.4)$ & $<0.001$ \\
\hline Stiff neck & $6(1.7)$ & $4(4.0)$ & $4(5.8)$ & 0.032 \\
\hline Wheezing & $2(0.6)$ & $6(6.0)$ & $7(10.1)$ & $<0.001$ \\
\hline Crepitation & $13(3.6)$ & $18(18.0)$ & $25(36.2)$ & $<0.001$ \\
\hline Dyspnea & $7(1.9)$ & $7(7.0)$ & $25(36.2)$ & $<0.001$ \\
\hline Respiration (/min), median (IQR) & $20(20-26)$ & $22(20-32)$ & $22(20-26)$ & 0.058 \\
\hline Body temperature $\left({ }^{\circ} \mathrm{C}\right)$, median (IQR) & $38.5(37.5-39.4)$ & $37.8(37.0-38.8)$ & $37.2(37.0-38.0)$ & $<0.001$ \\
\hline \multicolumn{5}{|l|}{ Hemodynamic profiles } \\
\hline SBP (mmHg), median (IQR) & $100(100-110)$ & $95(80-110)$ & $100(80-110)$ & $<0.001$ \\
\hline DBP (mmHg), median (IQR) & $60(60-70)$ & $60(50-70)$ & $60(50-70)$ & $<0.001$ \\
\hline Pulse (/min), median (IQR) & $100(86-120)$ & $100(89-115)$ & $102(90-124)$ & 0.049 \\
\hline
\end{tabular}

Note: $P$-values from nonparametric trend test across ordered groups.

Abbreviations: IQR, interquartile range; SBP, systolic blood pressure; DBP, diastolic blood pressure. 
Table 2 Laboratory profiles $(n=526)$

\begin{tabular}{|c|c|c|c|c|}
\hline \multirow[t]{2}{*}{ Characteristics } & \multirow{2}{*}{$\begin{array}{l}\text { Nonsevere }(n=357) \\
\text { Median (IQR) }\end{array}$} & \multirow{2}{*}{$\begin{array}{l}\text { Severe }(n=100) \\
\text { Median (IQR) }\end{array}$} & \multirow{2}{*}{$\begin{array}{l}\text { Dead }(n=69) \\
\text { Median (IQR) }\end{array}$} & \multirow[t]{2}{*}{$P$-value } \\
\hline & & & & \\
\hline \multicolumn{5}{|l|}{ Hematological } \\
\hline Hemoglobin, g/dL & $12.0(\mid 0.7-13.3)$ & $11.6(10.4-12.9)$ & I2.I (|0.2-|4.I) & 0.999 \\
\hline Hematocrit, \% & $35.8(31.8-39.6)$ & $34.0(30.4-38.8)$ & $36.4(30.6-42.1)$ & 0.805 \\
\hline Platelet count, $\times 1,000 / \mathrm{mm}^{3}$ & $117.0(72.0-196.0)$ & $72.0(42.5-143.5)$ & $50.0(2.8-85.8)$ & $<0.001$ \\
\hline WBC count, $\times 1,000 / \mathrm{mm}^{3}$ & $7.7(5.5-\mid I .2)$ & $10.45(7.5-6.0)$ & $12.0(8.8-6.1)$ & $<0.001$ \\
\hline Neutrophils (\%) & $68.0(56.0-79.8)$ & $82.8(68.3-88.1)$ & $84.0(75.7-89.0)$ & $<0.001$ \\
\hline Lymphocytes (\%) & $22.0(13.0-33.8)$ & II.I (6.8-20.0) & $8.1(4.9-14.9)$ & $<0.001$ \\
\hline Monocytes (\%) & $6.0(3.0-8.6)$ & $3.8(2.0-6.0)$ & $3.4(2.0-6.0)$ & $<0.001$ \\
\hline \multicolumn{5}{|l|}{ Biochemistry } \\
\hline Total bilirubin (mg/dL) & $0.7(0.5-1.3)$ & $2.4(0.8-5.7)$ & $4.4(2.4-6.6)$ & $<0.001$ \\
\hline Direct bilirubin (mg/dL) & $0.2(0.1-0.5)$ & I.I $(0.2-3.1)$ & $2.7(1.5-4.4)$ & $<0.001$ \\
\hline Albumin $(\mathrm{g} / \mathrm{dL})$ & $3.2(2.7-3.6)$ & $2.7(2.3-3.0)$ & $2.4(2.1-3.0)$ & $<0.001$ \\
\hline Globulin (g/dL) & $3.3(2.8-3.7)$ & $3.0(2.7-3.4)$ & $3.2(2.7-3.9)$ & 0.199 \\
\hline $\mathrm{ALT}(\mathrm{IU} / \mathrm{L})$ & $67.0(38.0-112.5)$ & $86.5(47.0-158.0)$ & $99.5(51.0-146.5)$ & $<0.001$ \\
\hline AST (IU/L) & $103.0(6 \mid .5-179.0)$ & $174.5(77.0-300.0)$ & $274.0(\mid 28.5-403.0)$ & $<0.001$ \\
\hline ALP (IU/L) & 165.5 (I20.5-267.5) & $27.6(141.0-416.0)$ & $243.0(128.0-342.0)$ & $<0.001$ \\
\hline BUN (mg/dL) & $12.0(9.0-17.0)$ & $31.0(14.0-54.0)$ & $55.5(27.0-72.0)$ & $<0.001$ \\
\hline Creatinine $(\mathrm{mg} / \mathrm{dL})$ & $0.9(0.7-1.2)$ & $1.4(0.9-2.5)$ & $2.5(1.5-5.0)$ & $<0.001$ \\
\hline $\mathrm{Na}(\mathrm{mmol} / \mathrm{L})$ & $134(131-137)$ & $133(129-136)$ & $132(129-137)$ & 0.034 \\
\hline $\mathrm{K}(\mathrm{mmol} / \mathrm{L})$ & $3.5(3.2-3.9)$ & $3.6(3.2-3.8)$ & $3.7(3.2-4.3)$ & 0.060 \\
\hline $\mathrm{Cl}_{2}(\mathrm{mmol} / \mathrm{L})$ & 101 (98-104) & $102(97-104)$ & $100(97-105)$ & 0.365 \\
\hline $\mathrm{CO}_{2}(\mathrm{mmol} / \mathrm{L})$ & $23(20-25)$ & $20(17-23)$ & $16(12-20)$ & $<0.001$ \\
\hline \multicolumn{5}{|l|}{ Urine analysis } \\
\hline \multicolumn{5}{|l|}{ Urine albumin, n (\%) } \\
\hline$<\mathrm{I}+$ & $190(64.0)$ & $40(47.1)$ & $12(19.7)$ & $<0.001$ \\
\hline$\geq 1+$ & $107(36.0)$ & $45(52.9)$ & $49(80.3)$ & \\
\hline \multicolumn{5}{|l|}{ Urine sugar, n (\%) } \\
\hline$<\mathrm{I}+$ & $292(98.3)$ & $84(98.8)$ & $59(96.7)$ & 0.535 \\
\hline$\geq 1+$ & $5(1.7)$ & $\mathrm{I}(\mathrm{I} .2)$ & $2(3.3)$ & \\
\hline
\end{tabular}

Abbreviations: IQR, interquartile range; WBC, white blood cell; ALT, alanine aminotransferase; AST, aspartate aminotransferase; ALP, alkaline phosphatase; BUN, blood urea nitrogen.

AST $>160$ IU/L (OR 2.89, 95\% CI 1.89-4.43), serum albumin $\leq 3.0 \mathrm{~g} / \mathrm{dL}$ (OR 4.69, 95\% CI 2.95-7.45), and serum creatinine $>1.4 \mathrm{mg} / \mathrm{dL}$ (OR 8.19, 95\% CI 5.06-13.35) (Table 3). Multicollinearity among variables was tested and was insignificant.

\section{The scoring system}

Item scores were derived by dividing the coefficient of significant predictors with the smallest coefficient (1.06) and rounding up to the nearest discrete number. The item scores varied from the minimum of 0 to the maximum of 4 .

Table 3 Significant predictors and assigned item score

\begin{tabular}{|c|c|c|c|c|c|c|}
\hline Predictors & Category & OR & $95 \% \mathrm{Cl}$ & $P$-value & Coefficient & Score \\
\hline \multirow[t]{2}{*}{ Age (years) } & $>15$ & 4.09 & $2.26-7.40$ & $<0.001$ & $\mathrm{I} .4 \mathrm{I}$ & 3 \\
\hline & $\leq 15$ & Ref & & & & \\
\hline \multirow[t]{2}{*}{ Pulse rate $(/ \mathrm{min})$} & $>100$ & 3.19 & $1.87-5.43$ & $<0.001$ & 1.16 & 2 \\
\hline & $\leq 100$ & Ref & & & & \\
\hline \multirow[t]{2}{*}{ Crepitation } & Yes & 2.97 & $1.63-5.39$ & $<0.001$ & 1.09 & 2 \\
\hline & No & Ref & & & & \\
\hline \multirow[t]{2}{*}{ AST (IU/L) } & $>160$ & 2.89 & $1.89-4.43$ & $<0.001$ & 1.06 & 2 \\
\hline & $\leq 160$ & Ref & & & & \\
\hline \multirow[t]{2}{*}{ Serum albumin $(\mathrm{g} / \mathrm{dL})$} & $\leq 3.0$ & 4.69 & $2.95-7.45$ & $<0.001$ & 1.55 & 3 \\
\hline & $>3.0$ & Ref & & & & \\
\hline \multirow[t]{2}{*}{ Serum creatinine (mg/dL) } & $>1.4$ & 8.19 & $5.06-13.25$ & $<0.001$ & 2.10 & 4 \\
\hline & $\leq \mathrm{I} .4$ & Ref & & & & \\
\hline
\end{tabular}

Notes: Coefficients from multivariable ordinal continuation ratio logistic regression.

Abbreviations: $\mathrm{OR}$, odds ratio; $\mathrm{Cl}$, confidence interval; $\mathrm{AST}$, aspartate aminotransferase. 
Each item scores were summed up to total scrub typhus severity score, which may vary from 0 to 16 (Table 3 ).

\section{Discrimination}

The mean severity scores in the nonsevere, severe, and dead groups were $4.2 \pm 2.5,8.9 \pm 3.1$, and $11.4 \pm 2.8$ (Table 4 and Figure 1).

\section{Clinical predictions}

The scores discriminated patients with severe or fatal scrub typhus from those with nonsevere scrub typhus with highlevel validity (area under receiver operating characteristic curve (AuROC) 0.9148, 95\% CI 0.8886-0.9410). The corresponding values for discriminating the fatal from the severe and nonsevere groups were lower (AuROC 0.9122, 95\% CI 0.8823-0.9420) (Figure 2).

By classifying the severity scores into three risk levels nonsevere (scores $\leq 5$ ), severe (scores 6-9), and most severe or fatal (scores $\geq 10$ ) - the scores classified 266 out of 357 patients correctly in the nonsevere group, with underestimation by one level in twelve cases and no instances of underestimation by two levels. In the severe group, the score classified correctly 43 out of 100 patients, with 19 cases underestimated and 81 cases overestimated. In the dead group, classification was correct in 50 out of 69 cases, with overestimation by one level in 45 cases and overestimation by two levels in ten cases (Table 4 ).

\section{Discussion}

Although patients with early and nonsevere scrub typhus usually respond very well to prompt treatment, patients with complications have a poorer prognosis, and the disease may well be fatal for them. Researchers have attempted to develop scoring systems to predict infectious diseases or their severity to help clinical management. Examples were a dengue fever scoring system to differentiate dengue fever from rickettsial and Q fever, which was developed in Taiwan, ${ }^{19}$ decision-tree algorithms to differentiate and prognosticate dengue hemorrhagic fever, ${ }^{20}$ and a prediction rule to evaluate the risk for community-acquired pneumonia to help admission decisions. ${ }^{18}$ These prediction rules used parameters of epidemiologic, demographic, clinical signs and symptoms, or laboratory characteristics. They all shared similar limitations of relatively large numbers of parameters, complicated laboratory tests beyond routine investigations, or tests that may be unavailable in many settings. These restrictions limited clinical application in routine practice.

For scrub typhus, there have been no studies attempting to develop a clinical algorithm to forecast disease severity to assist clinical diagnosis, evaluation, or management. The present study proposed a simple scoring system based on routinely clinical parameters available in most health care settings.

Predictors proposed in this study were more or less similar to what have been proposed in previous studies, such as serum albumin $\leq 3 \mathrm{~g} / \mathrm{dL}^{21}$ and serum creatinine $>1.4 \mathrm{mg} / \mathrm{dL} .{ }^{17}$ Some parameters were similar but different in categorization, such as the patient age ${ }^{21,22}$ and AST level. ${ }^{17}$ Some parameters were reported but were not significant in the present study, such as WBC count, ${ }^{17}$ platelets, ${ }^{17}$ and bilirubin. ${ }^{17}$

The present scrub typhus severity score, which ranged from 0 to 16 could be classified into three levels to simulate three levels of disease severities. We proposed the following interpretations and guidelines.

Patients scoring 0-5 were categorized as "nonsevere scrub typhus". These patients may be managed as outpatients. Antirickettsial agents like doxycycline, chloramphenicol, or azithromycin should be prescribed ${ }^{23,24}$ and scheduled for follow-up.

Patients scoring 6-9 were categorized as "severe scrub typhus". These patients are at higher risk of complications, and should be admitted to hospital for close observation. Further investigation and additional intervention may

Table 4 Score-classified severity levels, criterion-classified severity levels, and risk-estimation validity

\begin{tabular}{|c|c|c|c|c|c|c|c|}
\hline \multirow{2}{*}{$\begin{array}{l}\text { Score-classified } \\
\text { severity levels }\end{array}$} & \multirow{2}{*}{$\begin{array}{l}\text { Score } \\
\text { range }\end{array}$} & \multicolumn{3}{|c|}{ Criterion-classified severity levels } & \multicolumn{3}{|c|}{ Risk-estimation validity* } \\
\hline & & $\begin{array}{l}\text { Nonsevere } \\
n=357\end{array}$ & $\begin{array}{l}\text { Severe } \\
n=100\end{array}$ & $\begin{array}{l}\text { Dead } \\
n=69\end{array}$ & $\begin{array}{l}\text { Over } \\
\text { (\%) }\end{array}$ & $\begin{array}{l}\text { Correct } \\
(\%)\end{array}$ & $\begin{array}{l}\text { Under } \\
\text { (\%) }\end{array}$ \\
\hline Mean $\pm S D$ & & $4.2 \pm 2.5$ & $8.9 \pm 3.1$ & II. $.4 \pm 2.8$ & & & \\
\hline IQR & & $2-6$ & $7-12$ & $9-14$ & & & \\
\hline Nonsevere $(n=278)$ & $\leq 5$ & 266 & 12 & 0 & - & 50.6 & 2.3 \\
\hline Severe $(n=143)$ & $6-9$ & 81 & 43 & 19 & 15.4 & 8.2 & 3.6 \\
\hline \multirow[t]{2}{*}{ Fatal $(n=105)$} & $\geq 10$ & 10 & 45 & 50 & 10.4 & 9.5 & - \\
\hline & & & & Total & 25.8 & 68.3 & 5.9 \\
\hline
\end{tabular}

Note: *Percentage of total patients.

Abbreviations: SD, standard deviation; IQR, interquartile range. 
Nonsevere

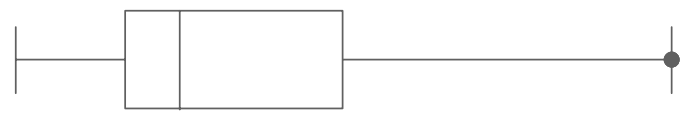

Severe

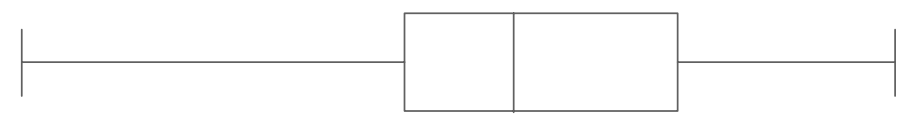

Dead

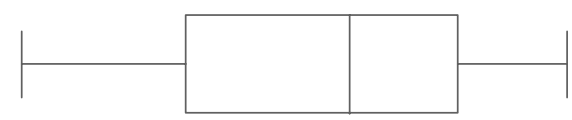

\begin{tabular}{llllllllllllllll|l}
\hline & 1 & 2 & 3 & 4 & 5 & 6 & 7 & 8 & 9 & 10 & 11 & 12 & 13 & 14 & 15 & 16 \\
& \multicolumn{1}{c}{ Scrub typhus severity scores }
\end{tabular}

Figure I Distribution of scrub typhus severity scores.

be needed. Patients encountered in lower-level hospitals might be referred to better-equipped hospitals.

Patients scoring 10 or above were classified as "fatal scrub typhus". These patients experienced the highest risk of death and should be fully investigated for system abnormalities or other life-threatening clinical risks, and should be admitted to an intensive care unit for close monitoring.

An overestimation (25.8\%) in the nonsevere group may have resulted in overzealous case management, but this may be considered beneficial for patients. Underestimation was observed in $3.6 \%$ of patients with severe scrub typhus. However, the same antirickettsial agents were routinely prescribed in these patients, and the clinical risk should therefore be considered acceptable.

The diagnosis of scrub typhus in the present study was defined as suspected cases based on World Health Organization criteria. This is the most practical definition used in poor, developing countries, where definite diagnosis like indirect immunofluorescence antibodies was hardly available in routine practice. We (the authors) believe that this definition, although not definite, is more relevant in the real world.

The present scoring system, like any other clinical prediction rules, should be validated by independent data before application to routine clinical practice.

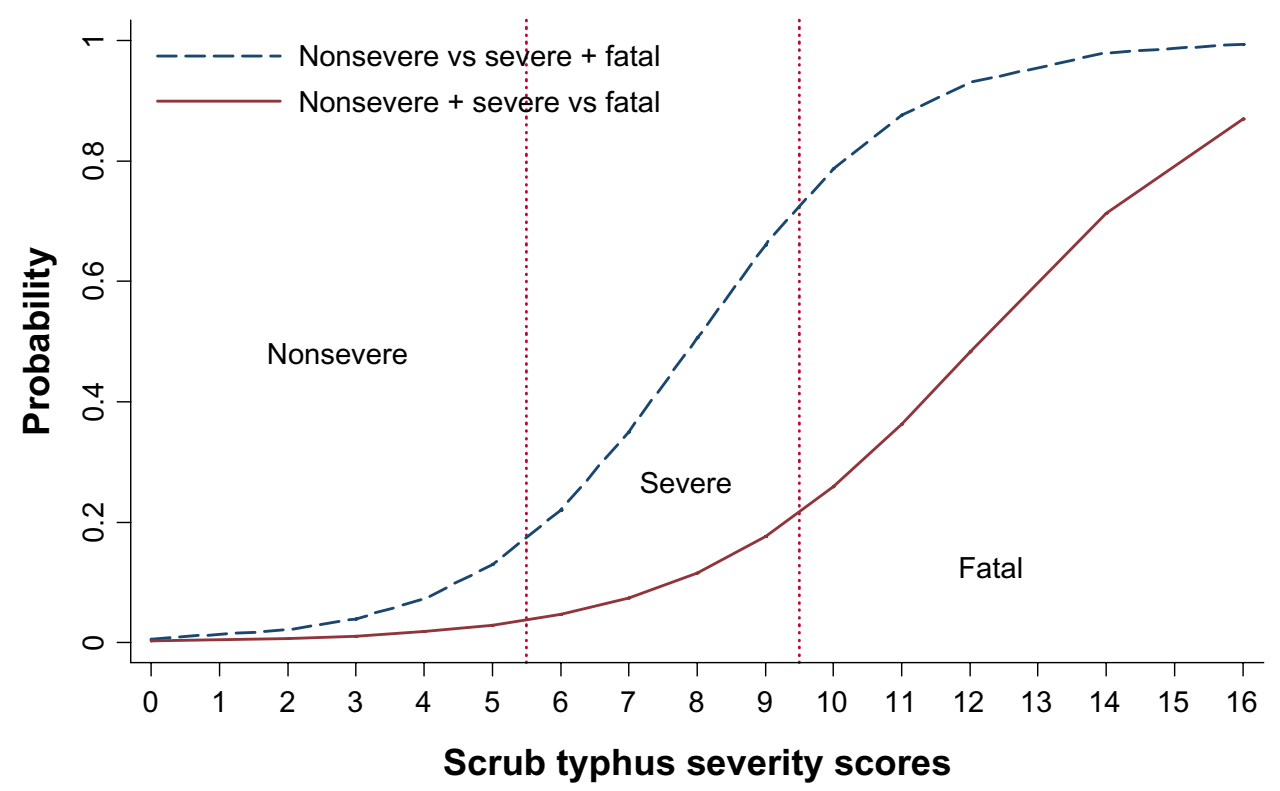

Figure 2 Discrimination of severity based on scrub typhus severity scores. 


\section{Conclusion}

The derived scrub typhus severity score classified patients into their severity levels with high levels of prediction, with clinically acceptable under- and overestimations. This classification may assist clinicians in patient prognostication, investigation, and management. Like other clinical prediction rules, the present scoring algorithm needs to be validated by independent data before being adopted into routine clinical practice.

\section{Acknowledgments}

The authors wish to thank the authorities of the two hospitals for their support. The study was partially funded by a grant from The Graduate School of Chiang Mai University, Chiang Mai, Thailand.

\section{Disclosure}

The authors report no conflicts of interest in this work.

\section{References}

1. Leelarasamee A, Chupaprawan C, Chenchittikul M, Udompanthurat S. Etiologies of acute undifferentiated febrile illness in Thailand. $J$ Med Assoc Thai. 2004;87(5):464-472.

2. Suttinont C, Losuwanaluk K, Niwatayakul K, et al. Causes of acute, undifferentiated, febrile illness in rural Thailand: results of a prospective observational study. Ann Trop Med Parasitol. 2006;100(4):363-370.

3. Moron CG, Popov VL, Feng HM, Wear D, Walker DH. Identification of the target cells of Orientiatsutsugamushi in human cases of scrub typhus. Mod Pathol. 2001;14(8):752-759.

4. Watt G, Kantipong P. Orientia tsutsugamushi and scrub typhus. In: Ruoult D, Parola P, editors. Rickettsial Diseases. New York: Information Healthcare; 2007:236-256.

5. Jeong YJ, Kim S, Wook YD, Lee JW, Kim KI, Lee SH. Scrub typhus: clinical, pathologic, and imaging findings. Radiographics. 2007;27(1):161-172.

6. Tsay RW, Chang FY. Serious complications in scrub typhus. J Microbiol Immunol Infect. 1998;31(4):240-244.

7. Wang CC, Liu SF, Liu JW, Chung YH, Su MC, Lin MC. Acute respiratory distress syndrome in scrub typhus. Am J Trop Med Hyg. 2007;76(6):1148-1152.

8. Chanta C, Triratanapa K, Ratanasirichup P, Mahaprom W. Hepatic dysfunction in pediatric scrub typhus: role of liver function test in diagnosis and marker of disease severity. J Med Assoc Thai. 2007;90(11): 2366-2369.
9. Kumar M, Krishnamurthy S, Delhikumar CG, Narayanan P, Biswal N, Srinivasan S. Scrub typhus in children at a tertiary hospital in southern India: clinical profile and complications. J Infect Public Health. 2012;5(1):82-88.

10. Rathi N, Rathi A. Rickettsial infections: Indian perspective. Indian Pediatr. 2010;47(2):157-164.

11. Yen TH, Chang CT, Lin JL, Jiang JR, Lee KF. Scrub typhus: a frequently overlooked cause of acute renal failure. Ren Fail. 2003;25(3): 397-410.

12. Jim WT, Chiu NC, Chan WT, et al. Clinical manifestations, laboratory findings and complications of pediatric scrub typhus in eastern Taiwan. Pediatr Neonatol. 2009;50(3):96-101.

13. Suputtamongkol Y, Suttinont C, Niwatayakul K, et al. Epidemiology and clinical aspects of rickettsioses in Thailand. Ann NY Acad Sci. 2009;1166:172-179.

14. World Health Organization. WHO recommended surveillance standards. Available from: http://www.who.int/csr/resources/publications/ surveillance/whocdscsrisr992.pdf. Accessed October 5, 2011.

15. Lee CS, Hwang JH, Lee HB, Kwon KS. Risk factors leading to fatal outcome in scrub typhus patients. Am J Trop Med Hyg. 2009;81(3): 484-488.

16. Thap LC, Supanaranond W, Treeprasertsuk S, Kitvatanachai S, Chinprasatsak S, Phonrat B. Septic shock secondary to scrub typhus: characteristics and complications. Southeast Asian J Trop Med Public Health. 2002;33(4):780-786.

17. Varghese GM, Abraham OC, Mathai D, et al. Scrub typhus among hospitalised patients with febrile illness in South India: magnitude and clinical predictors. $J$ Infect. 2006;52(1):56-60.

18. Fine MJ, Auble TE, Yealy DM, et al. A prediction rule to identify lowrisk patients with community-acquired pneumonia. $N$ Engl J Med. 1997;336(4):243-250.

19. Chang K, Lu PL, Ko WC, et al. Dengue fever scoring system: new strategy for the early detection of acute dengue virus infection in Taiwan. J Formos Med Assoc. 2009;108(11):879-885.

20. Tanner L, Schreiber M, Low JGH, et al. Decision tree algorithms predict the diagnosis and outcome of dengue fever in the early phase of illness. PLoS Negl Trop Dis. 2008;2(3):e196.

21. Kim DM, Kim SW, Choi SH, Yun NR. Clinical and laboratory findings associated with severe scrub typhus. BMC Infect Dis. 2010;10:108.

22. Wu KM, Wu ZW, Peng GQ, Wu JL, Lee SY. Radiologic pulmonary findings, clinical manifestations and serious complications in scrub typhus: experiences from a teaching hospital in eastern Taiwan. Int $J$ Gerontol. 2009;3(4):223-232.

23. Chrispal A, Boorugu H, Gopinath KG, et al. Scrub typhus: an unrecognized threat in South India - clinical profile and predictors of mortality. Trop Doct. 2010;40(3):129-133.

24. SuputtamongkolY. Scrub typhus. Poster presented at: 13th International Congress on Infectious Diseases; June 19-22, 2008; Kuala Lumpur, Malaysia.
Risk Management and Healthcare Policy

\section{Publish your work in this journal}

Risk Management and Healthcare Policy is an international, peerreviewed, open access journal focusing on all aspects of public health, policy, and preventative measures to promote good health and improve morbidity and mortality in the population. The journal welcomes submitted papers covering original research, basic science, clinical \& epidemio-

\section{Dovepress}

logical studies, reviews and evaluations, guidelines, expert opinion and commentary, case reports and extended reports. The manuscript management system is completely online and includes a very quick and fair peerreview system, which is all easy to use. Visit http://www.dovepress.com/ testimonials.php to read real quotes from published authors. 